Supporting Information for

\title{
Enhancement and Suppression of Resonance Energy Transfer Near Metal Nanoparticles
}

Yeonjun Jeong ${ }^{\dagger}$ and George C. Schatz ${ }^{*} \dagger$,

${ }^{\dagger}$ Department of Chemistry and Graduate Program in Applied Physics, Northwestern University, Evanston, Illinois 60208, United States
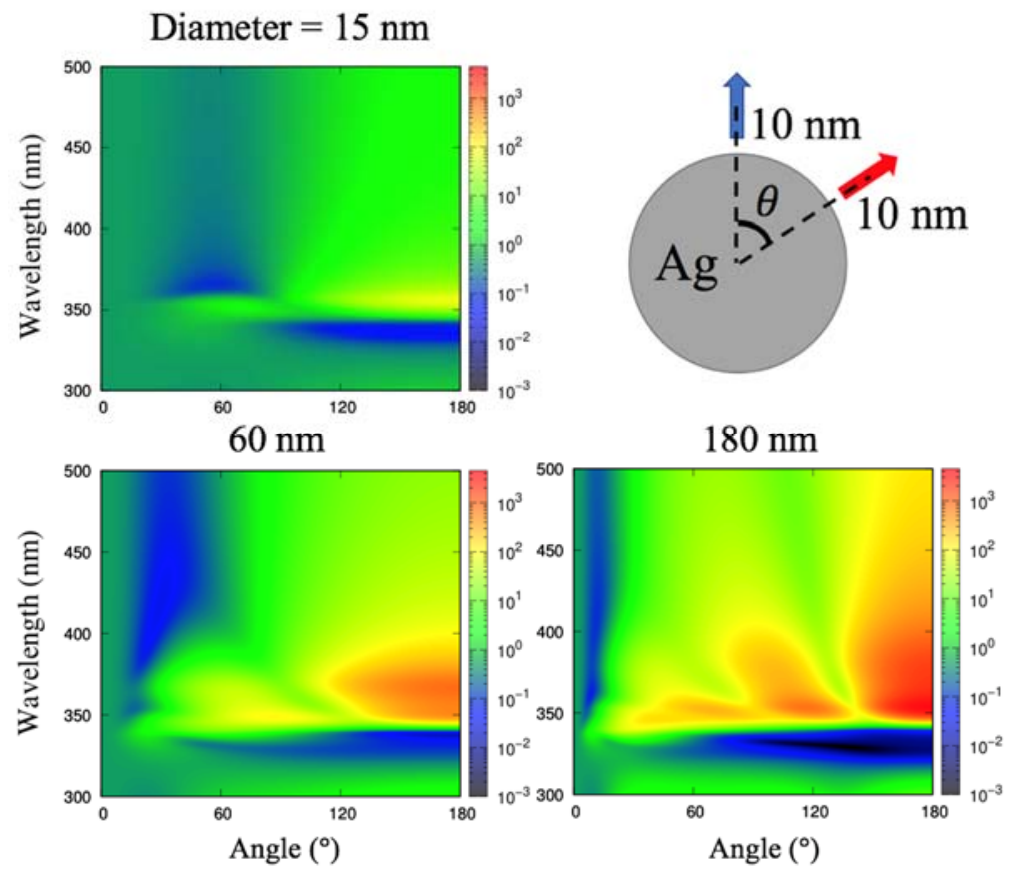

Figure S1. RET enhancement spectrum for radially oriented donor dipole (blue) and acceptor dipole (red) near a silver sphere as calculated with Mie theory. The dipole-sphere surface separation was kept at $10 \mathrm{~nm}$. 
(a)

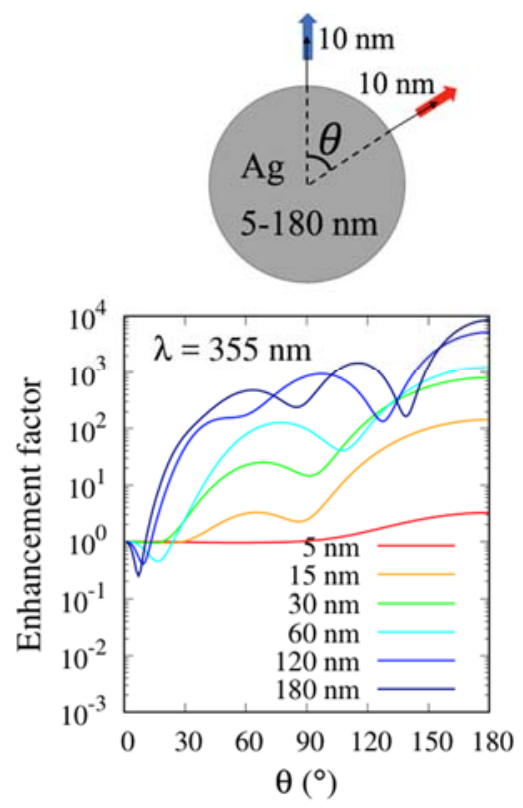

(b)
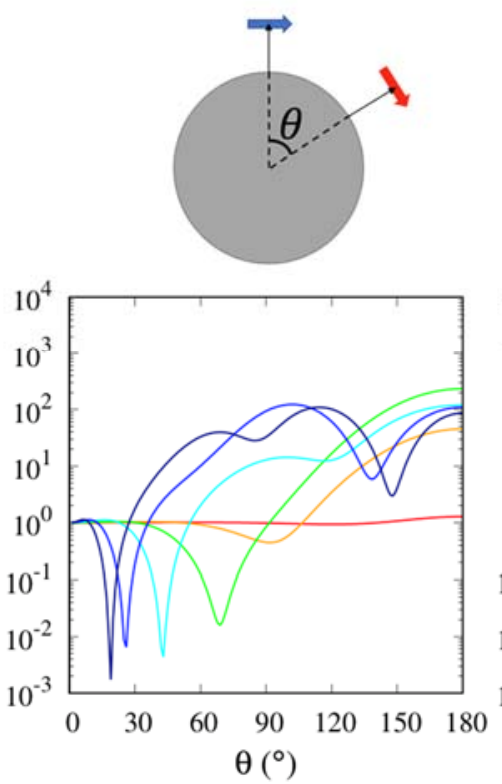

(c)
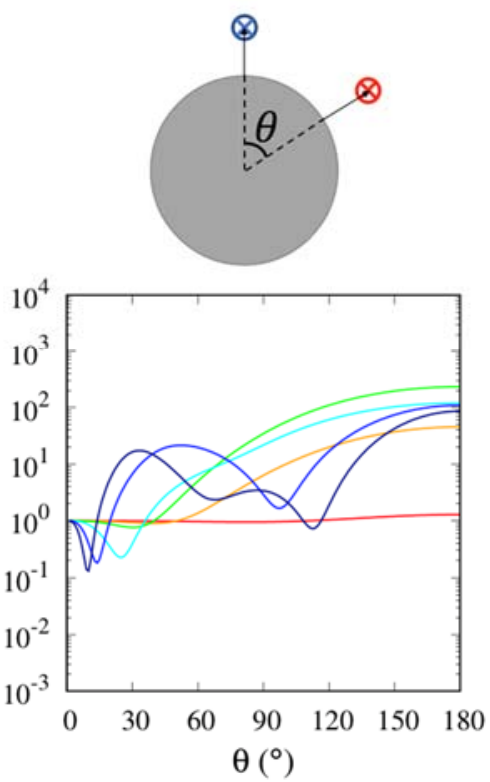

Figure S2. Plasmon enhancement factor for RET at $355 \mathrm{~nm}$ calculated for a silver sphere of varying sizes using Mie theory for three different orientations of the donor and the acceptor dipole versus position of the acceptor dipole represented as the angle between the donor and the acceptor dipole.

(a)

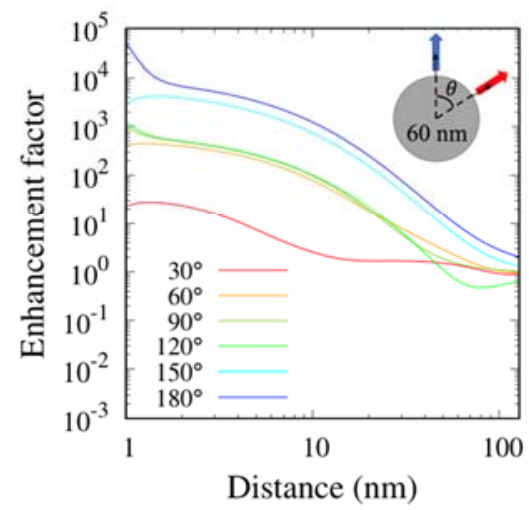

(b)

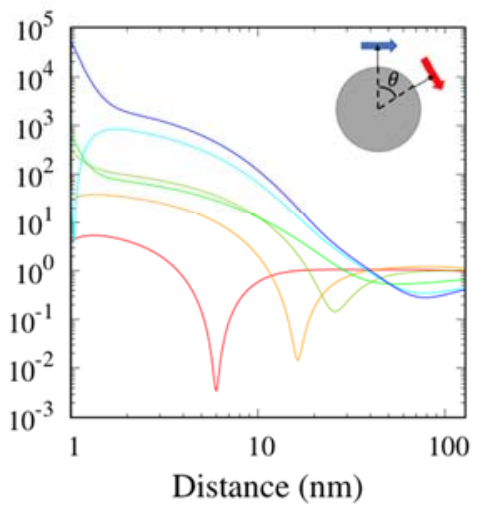

(c)

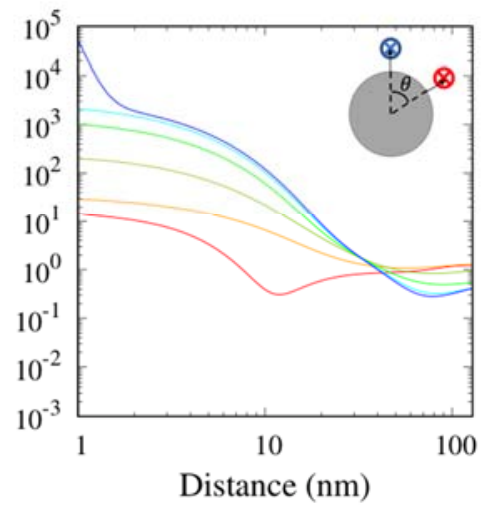

Figure S3. Dependence of the enhancement factor on the distance between the dipoles and the metal (silver) surface at various relative positions (angle) of the dipoles for three different dipole orientations. The donor and acceptor were equally separated from the surface, and the diameter of the sphere was $60 \mathrm{~nm}$. 
(a)

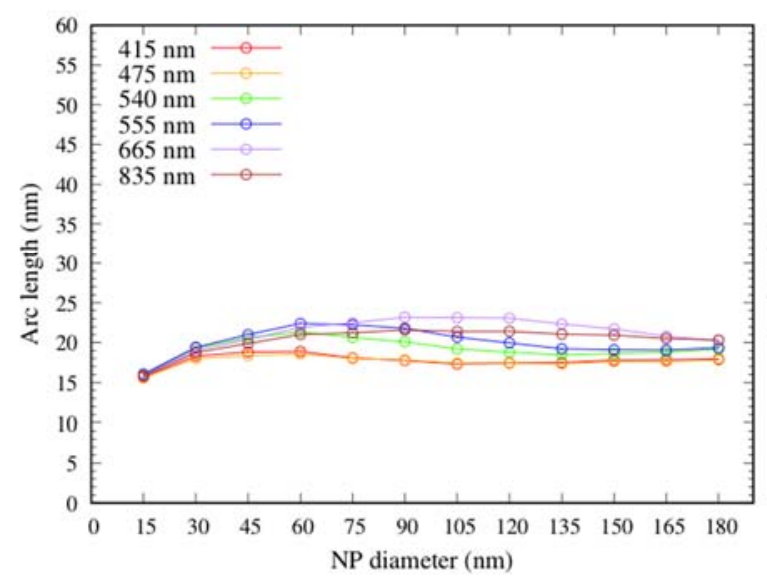

(b)

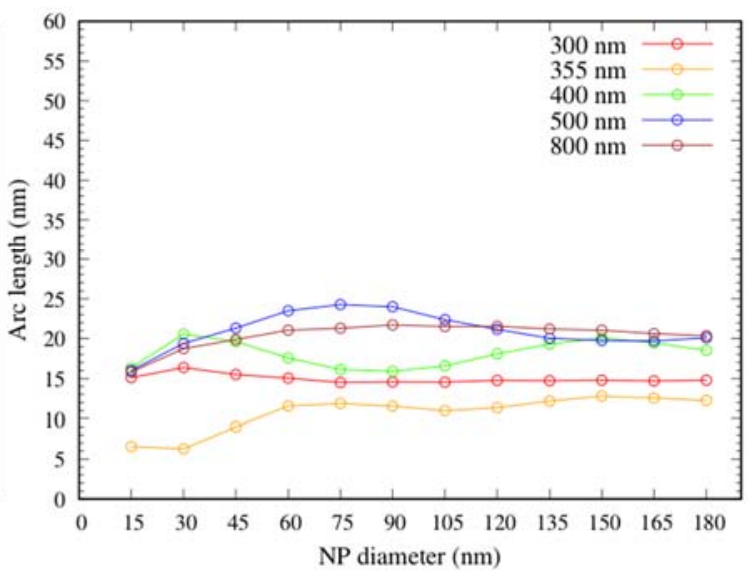

Figure S4. Arc length formed by the donor and acceptor at the minimum PEF point for varying NP sizes, where the NP material was (a) gold (b) silver, and the dipole-metal surface separation was 10 nm.

(a)

(b)

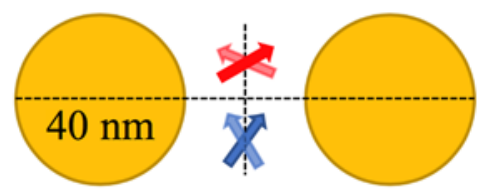

(c)
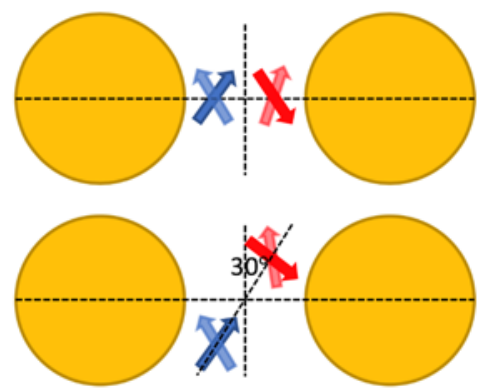

Figure S5. Three different geometrical setups of the RET simulation, termed (a) vertical (b) horizontal (c) diagonal in the text, derived from an experiment by Bidault et al. ${ }^{1}$ The RET rates were calculated by taking an average over 114 orientations that were uniformly distributed over a unit sphere for each dipole (total 12996 orientation sets). 
Table S1. Calculated donor absorption enhancement factor with a gold sphere for radial and tangential dipole orientations at dipole separation of $10 \mathrm{~nm}$ from the gold surface.

\begin{tabular}{|l|l|l|}
\hline \multicolumn{1}{|c|}{ Dipole orientation } & Radial & Tangential \\
\hline 5 & & \\
\hline 15 & 1.0535 & 0.9763 \\
\hline 30 & 1.6019 & 0.7553 \\
\hline 60 & 3.1197 & 0.4065 \\
\hline 120 & 7.4347 & 0.0701 \\
\hline 180 & 15.2486 & 0.0635 \\
\hline
\end{tabular}

\section{REFERENCES}

1. Bidault, S.; Devilez, A.; Ghenuche, P.; Stout, B.; Bonod, N.; Wenger, J., Competition between Förster Resonance Energy Transfer and Donor Photodynamics in Plasmonic Dimer Nanoantennas. ACS Photonics 2016, 3, 895-903. 